\title{
CAPACIDAD DIAGNÓSTICA DEL HRT-II Y DE LAS PERIMETRÍAS TOP, PULSAR Y FDT EN PACIENTES SOSPECHOSOS DE SUFRIR GLAUCOMA
}

\section{DIAGNOSTIC CAPABILITY OF PULSAR, FDT Y HRT-II IN GLAUCOMA SUSPECTS}

\author{
GONZÁLEZ-DE-LA-ROSA Mํ, GONZÁLEZ-HERNÁNDEZ Mํ, AGUILAR-ESTÉVEZ J², \\ DÍAZ-ALEMÁN T², ARMAS-PLASENCIA R ${ }^{3}$
}

\section{RESUMEN}

Objetivos: Determinar la capacidad diagnóstica de TOP-32, PULSAR-T30W, FDT-Umbral-N30 y HRT-II en glaucoma de sospecha.

Métodos: 47 ojos de 47 sujetos remitidos por sospecha de glaucoma (SG) se examinaron dos veces. Se excluyeron los casos con defecto medio (MD) superior a $6 \mathrm{~dB}$ en TOP-32. Los resultados se compararon con los obtenidos en 70 sujetos normales control (C).

Resultados: No se observaron diferencias significativas entre los valores de MD obtenidos en TOP32 , en los grupos $\mathrm{SG}(0,96 \mathrm{~dB}$. $\mathrm{DE}=1,7)$ y $\mathrm{C}(0,8 \mathrm{~dB}$. $\mathrm{DE}=1,77)(\mathrm{p}>0.05)$. El área papilar de SG $(2,12$ $\left.\mathrm{mm}^{2} . \mathrm{DE}=0.34\right)$ fue significativamente superior que en $C\left(1,97 \mathrm{~mm}^{2}\right.$. DE $\left.=0,45\right)(\mathrm{p}<0,01)$. Para una especificidad del $95 \%$, la raíz cuadrada de la varianza de pérdida (sLV) de PULSAR presentó la mayor sensibilidad $(30,9 \%)$ en exámenes individuales. La mayor reproducibilidad diagnóstica se obtuvo con la máxima elevación del contorno de HRT-II (23\%) y con el Plano de referencia $(23,4 \%)$, siendo del 14,9\% para varios índices, después de corregir la

\section{ABSTRACT}

Purpose: To determine the diagnostic capability of PULSAR-T30W, FDT-Threshold-N30 and HRT-II in glaucoma suspects.

Methods: Forty-seven eyes from 47 referred glaucoma suspects (GS) were examined twice with each technique. Cases with TOP-WW-MD $>6 \mathrm{~dB}$ were excluded. Results were compared with those of 70 eyes from 70 normal controls (C).

Results: Mean MD value using TOP-WW in the GS group $(0.96 \mathrm{~dB}$. sd=1.7) was not significantly different from $\mathrm{C}(0.8 \mathrm{~dB} . \mathrm{sd}=1.77)(\mathrm{p}>0.05)$. Disc area in GS group $\left(2.12 \mathrm{~mm}^{2}\right.$. sd $\left.=0.34\right)$ was significantly greater than in $\mathrm{C}\left(1.97 \mathrm{~mm}^{2}\right.$. $\left.\mathrm{sd}=0.45\right)$ $(\mathrm{p}<0.01)$. For $95.7 \%$ specificity, PULSAR-sLV showed the highest sensitivity of $30.9 \%$ in individual examinations. The highest reproducible sensitivity in the two examinations was obtained using HRT-II maximum contour elevation $(23.4 \%)$ and reference height (23.4\%), and was $14.9 \%$ for various indices after correcting for the influence of disc area (cup area, cup/disc area ratio, maximum contour depression and mean RNFL thickness). Reproducible sen-

\footnotetext{
Recibido: 31/5/06. Aceptado: 12/6/07.

Servicio de Oftalmología. Hospital Universitario de Canarias. Universidad de La Laguna. Islas Canarias. España.

1 Doctor en Medicina.

2 Licenciado en Medicina.

3 Diplomado en Óptica.

El primer autor tiene interés comercial en el programa TOP y en el perímetro PULSAR usados en este trabajo.

Financiado en parte por el Fondo de Investigación Sanitaria (FIS). Instituto Carlos III. Ministerio de Sanidad y Consumo.
}

Correspondencia:

Manuel González de la Rosa

C/. 25 de Julio, 34

38004 Santa Cruz de Tenerife

España

E-mail: mgdelarosa@jet.es 
influencia del tamaño papilar (área de la excavación, cocientes de área excavación/papila, máxima depresión del contorno y espesor de la capa de fibras). La reproducibilidad de los índices perimétricos fue: PULSAR-MD=8,5\%, PULSAR$\mathrm{sLV}=17 \%$, FDT-MD=6,4\%, FDT-PDT $=4,3 \%$. La asociación entre índices perimétricos y HRT-II elevó la sensibilidad pero redujo la reproducibilidad diagnóstica.

Conclusiones: Los índices de mayor eficacia fueron la máxima elevación del contorno, el plano de referencia y PULSAR-sLV, aunque la inclusión del aspecto papilar en la selección del grupo SG favoreció los resultados de HRT-II.

Palabras clave: Glaucoma, perimetría, campo visual, diagnóstico, nervio óptico. sitivity of the perimetric indices was: PULSAR$\mathrm{MD}=8.5 \%$, PULSAR-sLV=17\%, FDT-MD=6.4\%, FDT-PSD $=4.3 \%$. The association of perimetric and HRT-II indices achieved high sensitivity but low diagnostic reproducibility.

Conclusions: The most effective indices were maximum contour elevation, reference height and PULSAR-sLV, although the inclusion of the optic nerve head assessment in the selection of the GS sample may have favored the HRT-II results (Arch Soc Esp Oftalmol 2007; 82: 413-422).

Key words: Glaucoma, perimetry, visual field, diagnosis, optic nerve.

\section{INTRODUCCIÓN}

Drance (1) advierte que el diagnóstico de que no existe lesión alguna es mucho más complejo de lo que nuestras nociones corrientes de hipertensión nos sugieren. Efectivamente, a medida que mejora nuestro conocimiento sobre la fisiopatología de la enfermedad y progresan nuestros medios diagnósticos, la frontera entre normalidad y glaucoma comienza a desplazarse. Si prescindimos de los criterios prácticos establecidos habitualmente, hemos de admitir que este límite no está claramente definido. Si lo estuviera, serían superfluos nuestros esfuerzos por descubrir nuevos procedimientos cada vez más sensibles y precoces.

Numerosos investigadores consideran que «hipertensión ocular» y «glaucoma» son conceptos suficientemente bien definidos como para no plantear ningún tipo de dudas. Tal idea llevaría implícita la inutilidad de trabajos como el que proponemos aquí. Nuestra hipótesis es que este criterio es, por el contrario, un inconveniente a la hora de abordar la investigación de nuevos procedimientos de diagnóstico precoz. Así por ejemplo la costumbre de establecer un patrón de comparación constituye un inconveniente mas que una ayuda en la estructuración e interpretación de muchos de estos trabajos. Resulta una evidencia que, si asumimos que existe un procedimiento diagnóstico que debe ser reconocido como patrón, en el caso de existir un procedimiento mejor, sus resultados serán infravalorados al ser comparados con los que proporciona aquel. Cuando se aplica un patrón, los procedimientos estadísticos habituales de análisis de sensibilidad y especificad solamente son relativamente ciertos si este procedimiento es más perfecto que el evaluado. En el caso contrario el análisis se convierte en un sofisma matemático.

De forma ideal, ninguno de los procedimientos evaluados debería intervenir en el proceso de selección. Nuestra intención ha sido analizar varios de los procedimientos de examen desarrollados en los últimos años, para estimar su capacidad de diagnóstico precoz, tratando de evitar el laberinto lógico que constituye la utilización de un patrón rígido y evitando, en lo posible, los sesgos en la selección previa.

En este trabajo hemos pretendido investigar la capacidad diagnóstica y reproducibilidad de una batería de exámenes aplicados a pacientes remitidos por sospecha de glaucoma.

\section{SUJETOS, MATERIAL Y MÉTODOS}

Todos los controles normales (Grupo C) y los sospechosos de glaucoma (SG) realizaron un examen oftalmológico completo, incluyendo:

- Historia familiar y personal relacionada con el glaucoma, tal como migraña, síndrome de Raynaud, hipo o hiper-tensión arterial, diabetes, enfermedades cardiovasculares, tabaquismo o medicación.

- Refracción y agudeza visual. 
- Examen de segmento anterior y gonioscopia.

- Presión intraocular (Tonómetro de Goldmann).

- Topografía del nervio óptico con laser confocal (HRT-II) (Heidelberg Engineering, GerhartHauptmann-Strasse 30.69221 Dossenheim, Alemania).

- Perimetría FDT (Umbral N30) (Carl Zeiss Pty Ltd, 114 Pyrmont Bridge Road, Camperdown, NSW, 2050).

- Perimetría convencional de umbral luminoso blanco-blanco (TOP «32»WW) (2) usando el perímetro Octopus 311 (HAAG-STREIT, Gartenstadtstrasse 10 CH-3098 Köniz. Berna. Suiza).

- Perimetría PULSAR (T30W) (Prototipo experimental Octopus. HAAG-STREIT, Gartenstadtstrasse 10 CH-3098 Köniz. Berna. Suiza).

- Examen de polo posterior en midriasis.

\section{Criterios de inclusión}

Los pacientes del grupo SG se reclutaron consecutivamente entre 07/03/2003 y 08/08/2003 en nuestra Sección de Glaucoma, remitidos por diferentes ambulatorios y profesionales. Lo fueron esencialmente en función de sus cifras de presión intraocular. Se incluyeron pacientes con y sin tratamiento hipotensor, con presiones límites asociadas a glaucoma asimétrico, historia familiar de glaucoma o papila sospechosa. La sospecha de glaucoma se estableció por el conjunto de todos los datos relevantes. Los centros que remitían a los pacientes no poseían perímetro o topógrafo papilar.

El grupo $\mathrm{C}$ estuvo constituido por voluntarios normales que acudieron, durante el mismo período, para refracción o acompañando a pacientes. El campo visual no se tuvo en cuenta para su inclusión.

\section{Criterios de exclusión}

Se excluyó a los sujetos con patología asociada, tratamiento que pudiese afectar a la visión o al campo visual, pérdida significativa de transparencia del cristalino, agudeza visual corregida inferior a 0,8 , pupila de diámetro inferior a $3 \mathrm{~mm}$ o elevados errores en los controles perimétricos. Se exigió cámara anterior abierta en el examen gonioscópico. Todos los sujetos tenían errores refractivos inferiores a 5 dioptrías de equivalente esférico y a dos de cilindro.
Para la selección del grupo SG tratamos de asegurarnos de que nuestra investigación quedase limitada a glaucomas precoces, excluyendo aquellos casos que presentaron valores perimétricos de defecto medio (MD) superiores a $6 \mathrm{~dB}$ en el primer examen. Se excluyeron los que sufrían alguna enfermedad o tomaban algún medicamento con influencia sobre la visión, y los que presentaban historia familiar de glaucoma o hipertensión ocular, o bien signos asociados a la enfermedad (migraña, síndrome de Raynaud, vasculopatías, etc).

Igualmente fueron excluidos los sujetos que no realizaron la totalidad de los exámenes.

\section{Técnicas}

Los procedimientos de examen con HRT-II, FDT y TOP son bien conocidos en la literatura. Por el contrario la perimetría PULSAR es un procedimiento experimental que necesita ser descrito someramente:

Un fotómetro digital colocado en una esquina de un monitor de 19 pulgadas Sansung SyncMaster 959NF (Sansung Electronics Co, 416 Maetan3Dong, Paldal-Gu, Suwon City, Kyungki-Do, Corea), informa de su luminancia a un computador vía USB. El computador regula periódicamente el brillo y contraste según una escala previamente establecida. De esta manera puede mostrarse una escala con los 256 niveles habituales en computación.

El estímulo consiste en una onda circular, que decrece en contraste hacia la periferia, similar a la produce una gota al caer sobre el agua. La iluminación de fondo es de 100asb y el estímulo oscila por encima y debajo del fondo, de manera que en su conjunto es iso-luminante con él (fig. 1). La duración del estímulo es de $500 \mathrm{msec}$ y el diámetro $5^{\circ}$. La onda del estímulo puede modularse en resolución especial (desde 0,5 a 6,3 ciclos por grado en una escala de 12 niveles logarítmicos), contraste (32 niveles logarítmicos entre 3 y 100\%), color (blanco, rojo, verde y azul), movimiento centrífugo (de 2 a 20 ciclos por segundo en una escala logarítmica de 11 niveles) o frecuencia temporal, oscilando en fase y contra-fase $(10,15$ ó $30 \mathrm{~Hz})$.

- Para resolución espacial, la escala se establece desde un valor mínimo de frecuencia espacial de 0.5 ciclos/grado, usando la fórmula siguiente: Resolución espacial $(\mathrm{dLog})=10 \times \log$ (Frecuencia Espacial/0.5). 


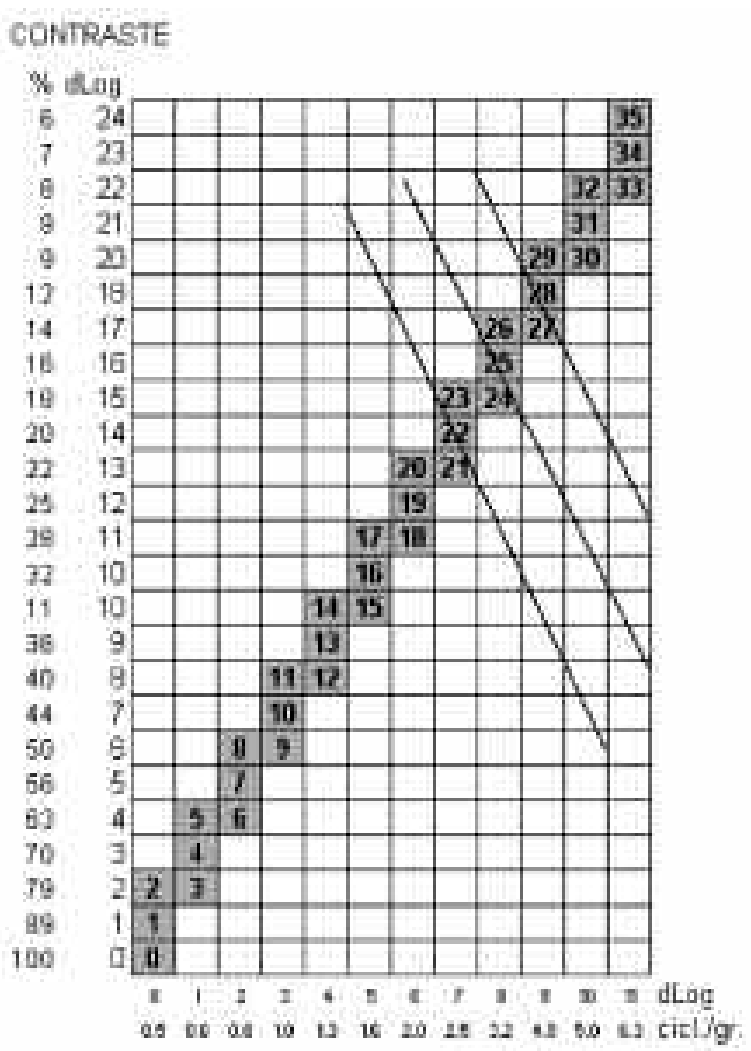

RESOLUCION ESPACIAL

Fig. 1: Escala de 36 niveles src, que combina frecuencias espaciales (sr) y contrastes $(C)$, y que es aproximadamente perpendicular a la porción descendente de la curva de sensibilidad al contraste para diferentes excentricidades.

- Como el contraste se sitúa por debajo y encima del fondo, se usó la siguiente fórmula: Contraste $=-20 \times \log$ (Amplitud central desde el fondo/intensidad del fondo).

$\mathrm{El}$ instrumento puede examinar diversas funciones visuales relacionadas con la Función de Sensibilidad al Contraste (Contrast Sensitivity Function o CSF) en el centro del campo visual, manteniendo constantes las restantes, para medir la sensibilidad de unas respecto a otras, usando la estrategia Tendency Oriented Perimetry (TOP). En base a estudios anteriores (3-5) se usó una escala de estímulos con 36 niveles en los que la resolución espacial (sr) y el contraste (c) varían simultáneamente (unidades src). Esta escala tiene la ventaja de ser aproximadamente perpendicular a las curvas de sensibilidad al contraste, las cuales son paralelas entre si en las diferentes regiones del campo visual (fig. 1). De esta manera se examina el umbral a mitad de la porción descendente de la curva hacia las altas frecuencias espaciales, donde ocurren preferentemente los defectos glaucomatosos, al menos para la visión central (6-8).

De la misma manera que para FDT (9), en PULSAR los umbrales se alteran cuando la refracción no es la adecuada [Fernandez-Baca G, González de la Rosa M, Perez Fernandez JR \& González Hernández M (2003): Influencia de la agudeza visual sobre la perimetría PULSAR T30W. 79 Congreso de la Sociedad Española de Oftalmología. Valencia]. Por ello, el instrumento muestra un test de agudeza visual para cerca antes del comienzo de cada examen. En el programa PULSAR T30W el estímulo se muestra en fase y contra-fase a $30 \mathrm{~Hz}$, lo que se ha demostrado útil en el diagnóstico precoz del glaucoma $(4,10-12)$.

En todos los casos, las dos primeras perimetrías se excluyeron para reducir el «efecto aprendizaje». Los tres exámenes perimétricos y el HRT-II se aplicaron dos veces en cada participante. Todos los exámenes se realizaron en un período de tiempo inferior al mes, con un descanso mínimo de 10 minutos. El campo visual se realizó con la refracción de lejos para FDT y TOP, y para cerca en PULSAR. La hipótesis de trabajo fue que la técnica diagnóstica más eficiente para el diagnóstico sería aquella que discriminase mejor entre ambos grupos SG y C, manteniendo alta especificidad.

Se usó regresión lineal para analizar la correlación entre los índices numéricos de cada técnica. La asociación entre índices perimétricos y anatómicos se evaluó usando regresión lineal y logarítmica. La probabilidad de que los índices resultasen diferentes de manera significativa entre los grupos SG y C se evaluó mediante T de Student. De evaluó igualmente una asociación entre índices obtenida previamente mediante análisis discriminante (11).

Se realizó un análisis ROC (Receiving Operating Characteristic), calculando las áreas ROC, intervalos de confianza, puntos de corte, sensibilidad, especificidad y probabilidad ( $\mathrm{T}$ de Student) para cada índice, usando un programa diseñado en Excel (Microsoft Corporation). Nuestro estudio se centró en el punto de corte que proporcionaba una especificidad más próxima al $95 \%$, puesto que un diagnóstico precoz exige una alta especificidad. El resultado de este programa fue verificado con el NCSS 2000 (NCSS, Kaysville, UT). Se consideró que el diagnóstico era reproducible cuando coinci- 
día en ambos exámenes. Por lo tanto, la reproducibilidad en el diagnóstico de anormalidad se calculó sobre el total de la nuestra, y no como un porcentaje de la sensibilidad. Para evaluar la reproducibilidad se analizó también el coeficiente de correlación intra-clases (13) usando el programa SPSS v. 12.0.1. (SPSS Inc. Chicago, ILL). El mismo programa se usó para calcular la potencia estadística de los resultados en relación al número de casos.

En el estudio se han respetado los principios de la Declaración de Helsinki.

\section{RESULTADOS}

Se excluyeron dos participantes del grupo $\mathrm{C}$ por mala colaboración y uno por no completar el segundo grupo de exámenes. Dos SG se excluyeron por cataratas, uno por baja colaboración, uno por no confirmarse la hipertensión ocular y dos por no completar el segundo grupo de exámenes.

Finalmente se estudiaron 70 ojos de 70 sujetos normales en el grupo C (promedio de edad 40,1 años, $\mathrm{DE}=16,5,39$ mujeres) y 47 ojos de 47 pacientes en el grupo SG (promedio de edad 60,4 años, $\mathrm{DE}=10,8,31$ mujeres). Todos los pacientes eran de raza caucásica excepto uno de raza negra perteneciente al grupo $\mathrm{SG}$.

En el grupo C, no se observó correlación entre la edad y ninguno de los índices analizados.

La duración de los exámenes perimétricos fue la siguiente: TOP $32 \mathrm{WW}=2: 52$ minutos $(\mathrm{DE}=0: 17$ ), PULSAR T30W= 3:50 minutos (DE=0:12) y FDT $\mathrm{N} 30=4: 31$ minutos $(\mathrm{DE}=0: 19)$.

El promedio de defecto medio (MD) al usar TOP 32 WW fue: Grupo $\mathrm{C}=0,80 \mathrm{~dB}(\mathrm{DE}=1.8)$ y grupo $\mathrm{SG}=0,96 \mathrm{~dB}(\mathrm{DE}=1,7)(\mathrm{p}>0,05)$ (fig. 2).

El promedio de raíz cuadrada de la varianza de pérdida (sLV) al usar TOP 32WW fue: Group $\mathrm{C}=$ $1,77 \mathrm{~dB}(\mathrm{sd}=0,68)$ y grupo $\mathrm{SG}=2,30 \mathrm{~dB}(\mathrm{sd}=1,19)$ $(\mathrm{p}<0,01)$.

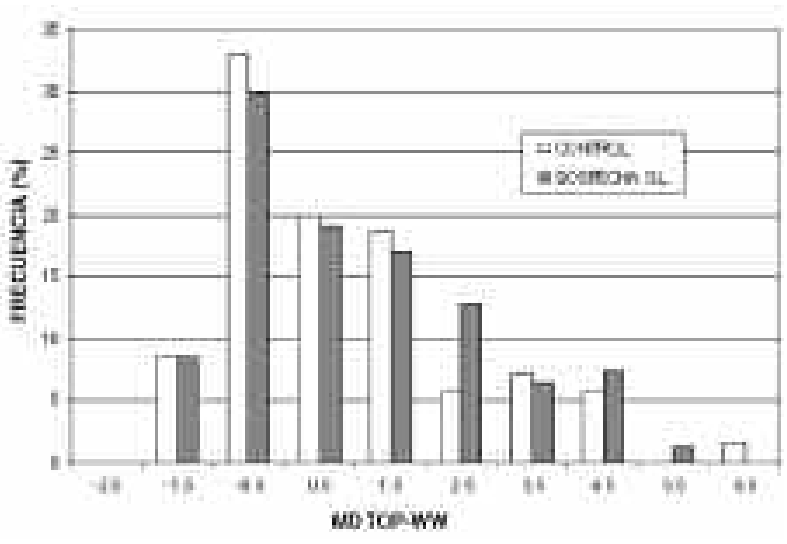

Fig. 2: Distribución del MD TOP WW en los pacientes control y glaucomas de sospecha.

El promedio de MD al usar PULSAR fue: Grupo $\mathrm{C}=0,13 \mathrm{~dB}(\mathrm{DE}=2,11)$ y grupo $\mathrm{SG}=1,25 \mathrm{~dB}$ $(\mathrm{DE}=2,72)(\mathrm{p}<0,01)$.

El promedio de la raíz cuadrada de la Varianza de pérdida (sLV) usando PULSAR fue: Grupo $\mathrm{C}=$ $2,25 \mathrm{~dB} \quad(\mathrm{DE}=0,58)$ y grupo $\mathrm{SG}=2,94 \mathrm{~dB}$ $(\mathrm{DE}=1,00)(\mathrm{p}<0,01)$.

El promedio de MD usando FDT fue: Grupo $\mathrm{C}=$ $-1,59 \mathrm{~dB} \quad(\mathrm{DE}=2,42)$ y grupo $\mathrm{SG}=-1,81 \mathrm{~dB}$ $(\mathrm{DE}=3,03)(\mathrm{p}>0,05)$.

El promedio de la desviación típica sobre el patrón (PSD) usando FDT fue: Grupo $\mathrm{C}=4,33 \mathrm{~dB}$ $(\mathrm{DE}=1,97)$ y grupo $\mathrm{SG}=4,50 \mathrm{~dB} \quad(\mathrm{DE}=2,28)$ (p>0,05). El 39\% de los sujetos del grupo C presentaron al menos un defecto en los cinco puntos centrales.

El promedio de área papilar usando HRT-II fue: Grupo $\mathrm{C}=1,97 \mathrm{~mm}^{2}(\mathrm{DE}=0.45)$ y grupo $\mathrm{SG}=2,12$ $\mathrm{mm}^{2}(\mathrm{DE}=0,34)(\mathrm{p}<0,01)$.

La tabla I muestra el coeficiente de correlación (r) entre los principales índices numéricos de los tres tipos de perimetría. La mayor correlación se observó entre los valores de MD y sLV-PSD de cada procedimiento, seguida de los valores de MD respectivos de PULSAR y FDT.

Tabla I. Coeficientes de correlación entre las tres perimetrías

\begin{tabular}{lcrrrr}
\hline & MD TOP WW & sLV TOP WW & MD PULSAR & \multicolumn{1}{c}{ sLV PULSAR } & MD FDT \\
\hline SLV TOP WW & $0,72(\mathrm{p}<0,01)$ & & & & \\
DM PULSAR & $0,11(\mathrm{p}<0,05)$ & $0,23(\mathrm{p}<0,01)$ & & & \\
SLV PULSAR & $-0,021(\mathrm{p}>0,05)$ & $0,06(\mathrm{p}>0,05)$ & $0,67(\mathrm{p}<0,01)$ & $-0,27(\mathrm{p}<0,01)$ & \\
MD FDT & $-0,220(\mathrm{p}<0,01)$ & $-0,25(\mathrm{p}<0,01)$ & $-0,36(\mathrm{p}<0,01)$ & $-0,59(\mathrm{p}<0,01)$ \\
PSD FDT & $0,14(\mathrm{p}>0,05)$ & $0,22(\mathrm{p}<0,01)$ & $0,25(\mathrm{p}<0,01)$ & $0,17(\mathrm{p}>0,05)$ & $-0,59)$ \\
\hline \hline
\end{tabular}


Usando regresión lineal, el MD de PULSAR presentó correlación significativa con 9 de los 23 índices principales del HRT-II, la PSD de FDT con tres índices, el MD de FDT con uno y el SLV de PULSAR con uno. La aplicación de regresión logarítmica no modificó estos resultados.

Muchos de los índices HRT-II presentaron una alta dependencia del tamaño de la papila. La figura 3 muestra los coeficientes de correlación y el valor de probabilidad en el grupo $\mathrm{C}$.

Se observó una asociación significativa entre la Máxima elevación del contorno (Maximun contour elevation) y el Plano de referencia (Referente Height), tanto en el grupo $\mathrm{C}(\mathrm{r}=0,41, \mathrm{p}=0,0004)$ como en el grupo $\mathrm{SG}(\mathrm{r}=0,38, \mathrm{p}=0,0002)$.

La figura 4 muestra que el mejor de los resultados para los exámenes perimétricos se obtuvo con la sLV de PULSAR . El percentil 95\% de las áreas ROC del MD FDT y PSD FDT fue inferior al percentil $5 \%$ de sLV PULSAR.

Se observó una alta sensibilidad $(43,6 \%)$ para la formula obtenida previamente mediante análisis discrimínate combinando algunos índices anatómicos y funcionales (11) [COMBINATION $=-4,95+$ $(\operatorname{sLV}$ TOP WW $* 1,09)+(\operatorname{sLV}$ PULSAR * 0,61 $)+$ (MD TOP WW * -0,33) + (Max. depr. cont. * $3,56)$. Sin embargo, la reproducibilidad fue muy baja $(12,8 \%)$.

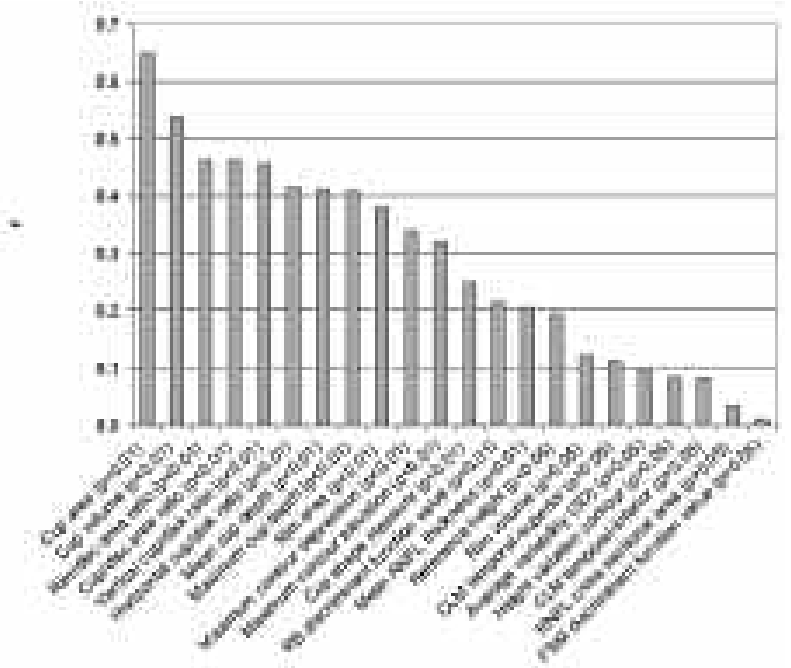

Fig. 3: Coeficiente de correlación ( $r$ ) y probabilidad $(p)$ entre el área papilar y el resto de los índices proporcionados por el HRT-II. Se ha conservado su nombre inglés, por no tener todos una traducción al castellano comúnmente aceptada.

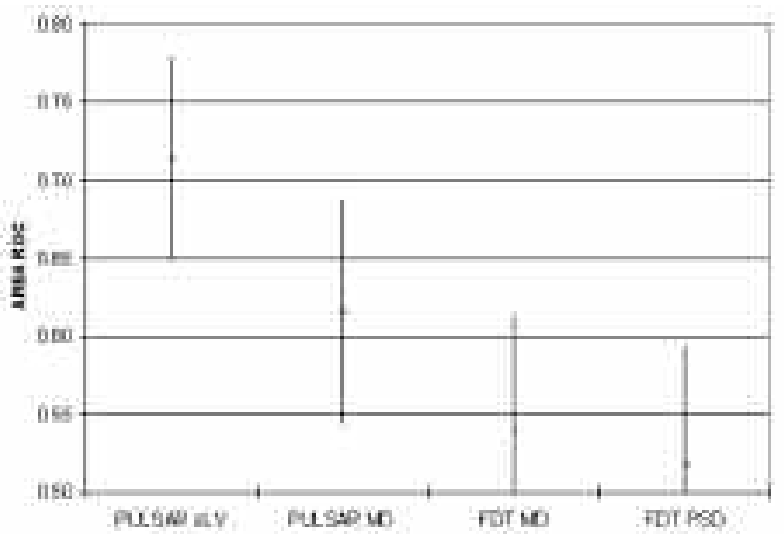

Fig. 4: Áreas ROC e intervalos de confianza de los índices perimétricos.

Se procedió a compensar algunos índices HRT II dependientes del tamaño papilar. Garway-Heath (14) y Jonas (15) han propuesto dos métodos para ello. Se calculó la ecuación de regresión entre cada índice y el tamaño papilar en la población control. La fórmula de la recta de regresión resultante sirvió para calcular el valor que debería alcanzar cada índice en cada paciente concreto en función del tamaño papilar. A continuación se calculó en los sujetos del grupo $\mathrm{SG}$, la desviación del valor real respecto al esperado en la población normal.

La tabla II muestra los resultados para una especificidad del 95,7\% (Intervalos de confianza 5\% y $95 \%=95,7 \pm 4,8 \%$ ). Se ha conservado el nombre inglés de los índices, por no tener todos una traducción al castellano comúnmente aceptada. El área excavación/papila y el conciente vertical excavación/papila se muestran en la tabla, con y sin corrección. La reproducibilidad indica el porcentaje de casos SG con diferencias significativas respecto al grupo $\mathrm{C}$ en los dos exámenes. Por ejemplo, para el índice Maximum contour elevation resultó anormal algo mas de la cuarta parte de los casos (sensibilidad $=26,6 \%$ como término medio en ambos exámenes), y en el $23,4 \%$ de la totalidad de los casos resultó anormal en ambos exámenes. La probabilidad (valor de $p$ ) del coeficiente de correlación intra-clases fue menor a 0,001 en todos los casos, excepto para la «Variabilidad promedio» (Average variability) $(\mathrm{p}=0,015)$ y «Combination» $(\mathrm{p}=0,629)$.

Cuando los dos exámenes perimétricos resultaron anormales, verificamos si ocurría lo mismo en ambos exámenes HRT-II. Esto ocurrió en tres casos de cuatro para el MD de PULSAR, seis de ocho 
Tabla II. Resultados

\begin{tabular}{|c|c|c|c|c|c|c|c|}
\hline Índice & $\begin{array}{l}\text { Área } \\
\text { ROC }\end{array}$ & $\mathrm{p}$ & Corte & Sens. & $\begin{array}{c}\text { Interv. Conf. } \\
\text { sens. }( \pm)\end{array}$ & Reprod. & $\begin{array}{l}\text { Coef. Corr. } \\
\text { intra-clases }\end{array}$ \\
\hline Maximum contour elevation & 65,3 & 0,00045 & 0,3 & 26,6 & 12,6 & 23,4 & 0,921 \\
\hline Reference height & 68,1 & 0,00026 & 0,5 & 28,7 & 12,9 & 23,4 & 0,778 \\
\hline PULSAR sLV & 71,3 & 0,00000 & 3,3 & 30,9 & 13,2 & 17,0 & 0,482 \\
\hline CDAR corrected with the Disk area & 61,5 & 0,00162 & 0,7 & 18,1 & 11,0 & 14,9 & 0,962 \\
\hline Cup area & 63,6 & 0,00128 & 2,7 & 14,9 & 10,2 & 14,9 & 0,942 \\
\hline Cup/disc area ratio (CDAR) & 60,1 & 0,00157 & 0,4 & 18,1 & 11,0 & 14,9 & 0,962 \\
\hline Maximum contour depression & 69,3 & 0,00004 & 0,4 & 17,0 & 10,7 & 14,9 & 0,578 \\
\hline Mean RNL thickness & 53,9 & 0,20772 & 0,2 & 17,0 & 10,7 & 14,9 & 0,827 \\
\hline Average variability & 66,1 & 0,00052 & 0,0 & 21,3 & 11,7 & 12,8 & 0,311 \\
\hline COMBINATION & 82,7 & 0,00000 & 0,3 & 43,6 & 14,2 & 12,8 & $-0,05$ \\
\hline Cup volume & 64,3 & 0,00075 & 0,8 & 16,0 & 10,5 & 12,8 & 0,974 \\
\hline Horizontal cup/disk ratio & 63,6 & 0,00060 & 1,0 & 18,1 & 11,0 & 12,8 & 0,804 \\
\hline Rim/disc area ratio & 60,7 & 0,00285 & 0,7 & 17,0 & 10,7 & 12,8 & 0,902 \\
\hline FSM discriminant function value & 49,9 & 0,21080 & $-0,2$ & 11,7 & 9,2 & 10,6 & 0,915 \\
\hline Height variation contour & 52,2 & 0,36699 & 0,6 & 16,0 & 10,5 & 10,6 & 0,571 \\
\hline Cup share measure & 58,8 & 0,01499 & 0,2 & 14,9 & 10,2 & 10,6 & 0,905 \\
\hline Maximum cup depth & 62,6 & 0,00124 & 1,0 & 16,0 & 10,5 & 10,6 & 0,881 \\
\hline Mean cup depth & 64,5 & 0,00020 & 0,4 & 16,0 & 10,5 & 8,5 & 0,828 \\
\hline PULSAR DM & 61,5 & 0,00224 & 3,9 & 14,9 & 10,2 & 8,5 & 0,753 \\
\hline $\mathrm{RB}$ discriminant function value & 51,3 & 0,34582 & 1,7 & 10,6 & 8,8 & 8,5 & 0,834 \\
\hline Vertical cup/disk ratio (VCDT) & 59,2 & 0,00919 & 0,6 & 22,3 & 11,9 & 8,5 & 0,955 \\
\hline VCDR corrected with the Disk area & 59,6 & 0,1481 & 1,1 & 8,5 & 8,0 & 8,5 & 0,955 \\
\hline FDT MD & 53,9 & 0,20290 & 5,3 & 11,7 & 9,2 & 6,4 & 0,731 \\
\hline Rim volume & 51,0 & 0,26485 & 0,0 & 7,4 & 7,5 & 6,4 & 0,855 \\
\hline CLM temporal-inferior & 54,3 & 0,28983 & 0,2 & 7,5 & 7,5 & 4,3 & 0,753 \\
\hline RNFL cross sectional area & 50,3 & 0,40125 & $-0,2$ & 7,4 & 7,5 & 4,3 & 0,821 \\
\hline FDT PSD & 51,8 & 0,24512 & 7,4 & 9,6 & 8,4 & 4,3 & 0,415 \\
\hline CLM temporal-superior & 48,5 & 0,49302 & 0,1 & 6,5 & 7,0 & 2,1 & 0,714 \\
\hline Rim area & 47,9 & 0,28966 & $-1,3$ & 2,1 & 4,1 & 2,1 & 0,902 \\
\hline
\end{tabular}

para SLV PULSAR, uno de tres para MD FDT y uno de dos para PSD de FDT.

El mayor área ROC correspondió a sLV PULSAR, pero se observó un importante solapamiento

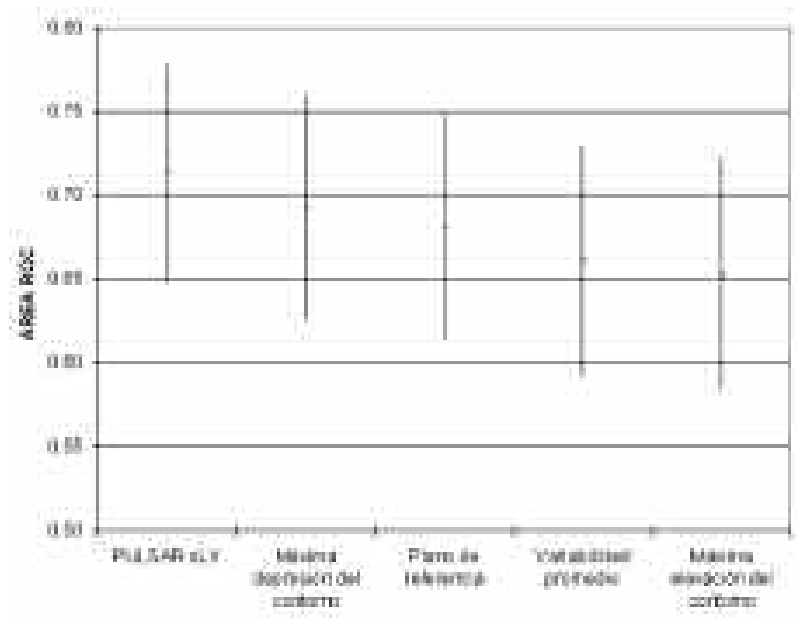

Fig. 5: Intervalos de confianza del área ROC de los mejores índices obtenidos. entre sus intervalos de confianza y los de otros de los mejores índices obtenidos (fig. 5).

Para distinguir entre las capacidades diagnósticas de los tres exámenes psicofísicos y los índices principales HRT-II (figs. 4 y 5), se calculó su potencia. Para los dos exámenes, comparando los grupos $\mathrm{C}$ y $\mathrm{SG}$, los valores de potencia fueron: MD de PULSAR $(0,727,0,652)$, sLV de PULSAR $(0,995,0,992)$, MD de FDT $(0,081,0,066)$, PSD de FDT $(0,060,0,189)$, Máxima depresión del contorno (Maximum contour depression) $(0,929,0,918)$, Máxima elevación del contorno $(0,836,0,776)$, Plano de referencia $(0,749$, $0,905)$ y Variabilidad promedio $(0,880,0,671)$.

\section{DISCUSIÓN}

\section{Discusión de los resultados funcionales}

Los valores medios de MD en TOP WW en nuestra muestra de SG son inferiores a los de la mayoría de los estudios realizados sobre glaucoma precoz 
(16-23) lo que explica que los resultados sean inferiores a otros estudios que han usado casos con defectos más profundos.

La selección de casos con MD TOP WW inferior a $6 \mathrm{~dB}$ en el primer examen, para el grupo SG, ha introducido un pequeño sesgo en los resultados de esta técnica respecto a las otras, en las que no se eliminaron los defectos más avanzados. El potencial diagnóstico de TOP WW puede haberse infravalorado, y por ello no se ha incluido en el estudio.

No hay razón para suponer que la diferencia de edad entre los grupos C y SG tenga influencia en los resultados. MD es un índice corregido por la edad, y nadie ha informado de influencias de la edad sobre sLV, PSD o los índices globales de HRT-II.

Por el contrario el presente trabajo confirma nuestros estudios anteriores que indican que PULSAR es sensible y específico para el diagnóstico precoz del glaucoma, así como nuestras observaciones previas sobre sLV. TOP calcula los umbrales tomando en consideración interrelaciones fisio-patológicas (24). Los estudios que han supuesto que la suavización del campo visual que produce y la reducción que induce en los valores de LV pueden conducir a una limitación diagnóstica, se basan en suposiciones (25), o en simulaciones usando escotomas aislados, sin relación con la fisio-patología del glaucoma (26).

La realidad es la opuesta; con TOP se observan pocos casos de LV elevada en sujetos normales. Los principales componentes de LV son la fluctuación del umbral, su irregularidad topográfica y los errores. Los errores se limitan en TOP al reducirse el «efecto fatiga» neurológico, las faltas de atención y los defectos metodológicos causados por examinar los puntos de forma independiente (27-29). Flammer (30) indicó que el primer signo de glaucoma no son los defectos focales o difusos sino el incremento de la fluctuación del umbral. Reduciendo los errores metodológicos, TOP parece detectar el incremento de la fluctuación como un defecto neuronal, probablemente previo o por lo menos independientemente de daño anatómico. PULSAR examina simultáneamente funciones de asociadas al contraste y frecuencias espaciales y temporales altas que podrían facilitar la detección de estos defectos funcionales.

\section{Discusión de los resultados morfológicos}

Si se tiene en cuenta el tamaño papilar, la sensibilidad diagnóstica de algunos índices HRT-II se reduce substancialmente. La proporción de esta reducción es variable y no afecta a la reproducibilidad diagnóstica, que continúa siendo alta. Algunos de ellos, como la Máxima elevación del contorno y el Plano de referencia alcanzaron la máxima capacidad diagnóstica. HRT-II calcula un valor específico para el Plano de referencia en cada examen, 50 micras por debajo de la línea de contorno en el sector temporal entre $350^{\circ}$ y $356^{\circ}$. Esto puede explicar el que se obtengan resultados comparables con ambos índices.

\section{Discusión de la relación entre función y morfología}

Respecto a los resultados obtenidos con HRT-II debemos reconocer que estudios anteriores (11) nos orientaron a pensar que su capacidad diagnóstica era inferior al valor de sLV en TOP-WW y a los índices PULSAR, pero esta opinión debe se revisada teniendo en cuenta los nuevos datos obtenidos respecto a la reproducibilidad diagnóstica y el coeficiente de correlación intra-cases de cada índice.

No ha podido evitarse un cierto grado de sesgo en la selección del grupo SG, puesto que los médicos que remitían al paciente conocían el aspecto oftalmoscópico de la papila. Por lo tanto, las diferencias entre los mejores índices anatómicos y perimétricos pueden ser circunstanciales y causadas por las características y tamaño de la muestra. Sin embargo, no hemos querido suprimir la información proporcionada por el HRT-II, para no excluir a los posibles glaucomas pre-perimétricos. La correlación intra-clases fue, en general, mayor para los índices HRT-II que para los perimétricos, en contradicción con los resultados de otros autores (31) que no han encontrado diferencias en la fluctuación a largo plazo de los índices perimétricos y HRT-II. Sin embargo el sesgo producido por la papilla en la selección del grupo SG y las diferencias de sensibilidad observadas entre PULSAR y los mejores índices HRT-II, plantean dudas respecto a la precocidad de los defectos morfológicos, puesto que las áreas ROC de HRT-II son menores y sus intervalos de confianza se solapan con los de sLV PULSAR.

Debemos añadir que la fórmula que habíamos obtenido en un trabajo previo mediante análisis discriminante (11) y que asocia varios índices perimétricos y HRT-II, ha confirmado en este trabajo su alta sensibilidad. Sin embargo, este hecho contrasta 
con una reproducibilidad diagnóstica bastante pobre, de manera que su habilidad diagnóstica global resulta inferior a la de los mejores índices perimétricos y HRT-II. Debemos concluir a la vista de esta nueva información que la asociación de índices perimétricos y papilares no proporciona tantas ventajas diagnósticas como habíamos supuesto.

La ausencia de diferencias entre la regresión lineal y la logarítmica podría estar en relación con las características de nuestra muestra: glaucoma incipiente, con escaso rango de defectos. Los MD de los tres tipos de perimetría presentaron alta correlación con el daño papilar, pero con menor capacidad diagnóstica que sLV PULSAR, que también alcanzó una correlación intra-clases inferior a MD. Esto podría indicar que sLV PULSAR proporciona una información pre-anatómica, mientras que MD estaría asociada a fases mas avanzadas del proceso patológico.

Los SG que se diferenciaron de los C por estas técnicas, no desarrollarán necesariamente glaucoma en el futuro. Esto es particularmente probable en muchos sospechosos con papilas grandes. Sin embargo debemos recordar que en el Ocular Hypertension Treatment Study $(32,33)$ el valor de PSD, que equivale en el perímetro Humphrey al sLV de los perímetros Octopus, parece superar a MD como factor de riesgo para desarrollar glaucoma.

La metodología de este trabajo ha sido similar a otro publicado recientemente por nuestro grupo, donde pueden consultarse algunas consideraciones adicionales respecto a la posibilidad de reducir los sesgos habituales en este tipo de estudios (34).

\section{BIBLIOGRAFÍA}

1. Drance SM. The early structural and functional disturbances of chronic open-angle glaucoma. Robert N. Shaffer lecture. Ophthalmology 1985; 92: 853-857.

2. Gonzalez de la Rosa M, Martinez, A, Sanchez M, Mesa C, Cordoves L, Losada MJ. Accuracy of the Tendency Oriented Perimetry with the Octopus 1-2-3 Perimeter. In: Wall M, Wild J. Perimetry Update 1996/1997. Amsterdam: Kugler Publ; 1997; 119-123.

3. Gonzalez-Hernandez M, Pareja Rios A, Rodriguez M, Gonzalez de la Rosa M. Combined Spatial Resolution and Contrast Perimetry in normal subjects. In: Wall M, Mills RP. Perimetry Update 2000/2001. Amsterdam: Kugler Publ; 2001; 109-114.

4. González-Hernández, M. Perimetrías de modulación temporal y movimiento. Dependencias respecto a la percepción del contraste y la resolución espacial. Aplicación al diagnóstico precoz del glaucoma. La Laguna: Universidad de La Laguna. 2002. Thesis doctoralis.
5.- Gonzalez-Hernandez, M, Fernandez-Vidal A, García-Feijoo J, Gonzalez de la Rosa M. Perimetric measurement of contrast sensitivity functions. In: Henson D, Wall M. Perimetry Update 2002/2003. Amsterdam: Kluger Pub; 2004; 109-114.

6. Arden GB, Jacobson JJ. A simple grating test for contrast sensitivity: preliminary results indicate value in screening for glaucoma. Invest Ophthalmol Vis Sci 1978; 17: 23-32.

7. Ross JE, Bron AJ, Clarke DD. Contrast sensitivity and visual disability in chronic simple glaucoma. Br J Opthalmol 1984; 68: 821-827.

8. Sample PA, Juang PS, Weinreb RN. Isolating the effects of primary open-angle glaucoma on the contrast sensitivity function. Am J Ophthalmol 1991; 112: 308-316.

9. Artes PH, Nicolela MT, McCormick TA, LeBlanc RP, Chauhan BC. Effects of blur and repeated testing on sensitivity estimates with frequency doubling perimetry. Invest Ophthalmol Vis Sci 2003; 44: 646-652.

10. Fernandez-Vidal AM, Garcia-Feijoo J, Garcia-Sanchez J. Pulsar perimetry: a new procedure for early glaucoma evaluation. In: Henson D, Wall M. Perimetry Update 2002/2003. Amsterdam: Kluger Pub; 2004; 199-205.

11. Gonzalez de la Rosa M, Mendez. Hernandez, C, Garcia Feijoo J, Garcia Sanchez J, Perez. Hernandez JR, Gonzalez-Hernandez M, et al. TOP strategy and Pulsar T30W perimetry in glaucoma early diagnosis. In: Reece SM. Focus on Glaucoma Research. New York: Nova Science Publishers Inc; 2005; 117-172.

12. Gonzalez de la Rosa M, Gonzalez-Hernandez M, Garcia Feijoo J, Morales J, Azuara-Blanco A. Diagnostic accuracy and reproducibility of tendency oriented perimetry in glaucoma. Eur J Ophthalmol 2006; 16: 259-267.

13. Bland JM, Altman DG. Measurement error and correlation coefficients. BMJ 1996; 313: 41-42.

14. Garway-Heath DF, Ruben ST, Viswanathan A, Hitchings $R A$. Vertical cup/disc ratio in relation to optic disc size: its value in the assessment of the glaucoma suspect. $\mathrm{Br} \mathrm{J}$ Ophthalmol 1998; 82:1118-1124.

15. Jonas JB, Bergua A, Schmitz-Valckenberg P, Papastathopoulos KI, Budde WM. Ranking of optic disc variables for detection of glaucomatous optic nerve damage. Invest Ophthalmol Vis Sci 2000; 41: 1764-1773.

16. Wollstein G, Garway-Heath DF, Hitchings RA. Identification of early glaucoma cases with the scanning laser ophthalmoscope. Ophthalmology 1998; 105: 1557-1563.

17. Fabre K, Michiels I, Zeyen T. The sensitivity and specificity of TOP, FDP and GDX in screening for early glaucoma. Bull Soc Belge Ophtalmol 2000; 275: 17-23.

18. Cello KE, Nelson-Quigg JM, Johnson CA. Frequency doubling technology perimetry for detection of glaucomatous visual field loss. Am J Ophthalmol 2000; 129: 314-322.

19. Wollstein G, Garway-Heath DF, Fontana L, Hitchings $R A$. Identifying early glaucomatous changes. Comparison between expert clinical assessment of optic disc photographs and confocal scanning ophthalmoscopy. Ophthalmology 2000; 107: 2272-2277.

20. Drasdo N, Aldebasi YH, Chiti Z, Mortlock KE, Morgan $J E$, North RV. The s-cone PHNR and pattern ERG in primary open angle glaucoma. Invest Ophthalmol Vis Sci 2001; 42: 1266-1272.

21. Zangwill LM, Bowd C, Berry CC, Williams J, Blumenthal EZ, Sanchez-Galeana CA, et al. Discriminating between 
normal and glaucomatous eyes using the Heidelberg Retina Tomograph, GDx Nerve Fiber Analyzer, and Optical Coherence Tomograph. Arch Ophthalmol 2001; 119: 985-993.

22. Bowd C, Zangwill LM, Berry CC, Blumenthal EZ, Vasile $C$, Sánchez-Galeana $C$, et al. Detecting early glaucoma by assessment of retinal nerve fiber layer thickness and visual function. Invest Ophthalmol Vis Sci 2001; 42: 1993-2003.

23. Chandrasekhar G, Kunjam V, Rao VS, Nutheti R. Humphrey visual field and frequency doubling perimetry in the diagnosis of early glaucoma. Indian J Ophthalmol 2003; 51: 35-38.

24. Gonzalez de la Rosa M, Gonzalez Hernandez M, Abraldes M, Azuara-Blanco A. Quantification of interpoint topographic correlations of threshold values in glaucomatous visual fields. J Glaucoma 2002; 11: 30-34.

25. Maeda $H$, Nakaura $M$, Negi A. New perimetric threshold test algorithm with dynamic strategy and tendency oriented perimetry (TOP) in glaucomatous eyes. Eye 2000; 14 : 747-751.

26. Anderson AJ. Spatial resolution of the tendency-oriented perimetry algorithm. Invest Ophthalmol Vis Sci 2003; 44: 1962-1968.

27. Gonzalez de la Rosa M, Arteaga V, Fernandez-Baca G, Gonzalez-Hernandez. M. Glaucoma diagnosis using Tendency Oriented Perimetry. In: Henson DB, Wall M. Perimetry Update 2002/2003. Amsterdam: Kugler Publ; 2004; 157-163.

28. Gonzalez-Hernandez M, Morales J, Azuara-Blanco A, Garcia Sanchez J, Gonzalez de la Rosa M. Comparison of diagnostic ability between a fast strategy, tendency oriented perimetry, and the standard bracketing strategy. Ophthalmologica 2005; 219: 373-378.

29. Gonzalez de la Rosa M, Gonzalez-Hernandez M, Garcia Feijoo J, Morales J, Azuara-Blanco A. Diagnostic accuracy and reproducibility of tendency oriented perimetry in glaucoma. Eur J Ophthalmol 2006; 16: 259-267.

30. Flammer J, Drance SM, Zulauf M. Differential light threshold. Short- and long-term fluctuation in patients with glaucoma, normal controls, and patients with suspected glaucoma. Arch Ophthalmol 1984; 102: 704-706.

31. Funk J, Mueller H. Comparison of long-term fluctuations: laser scanning tomography versus automated perimetry. Graefes Arch Clin Exp Ophthalmol 2003; 241: 721-724.

32. Kass MA, Heuer DK, Higginbotham EJ, Johnson CA, Keltner JL, Miller JP, et al. The Ocular Hypertension Treatment Study: a randomized trial determines that topical ocular hypotensive medication delays or prevents the onset of primary open-angle glaucoma. Arch Ophthalmol 2002; 120: 701-713.

33. Gordon MO, Beiser JA, Brandt JD, Heuer DK, Higginbotham EJ, Johnson CA, et al. The Ocular Hypertension Treatment Study: baseline factors that predict the onset of primary open-angle glaucoma. Arch Ophthalmol 2002; 120: 714-720.

34. de la Rosa MG, Gonzalez-Hernandez M, Lozano-Lopez V, Mendez MS, de la Vega RR. Optic disc tomography and perimetry in controls, glaucoma suspects, and early and established glaucomas. Optom Vis Sci 2007; 84: 33-41. 